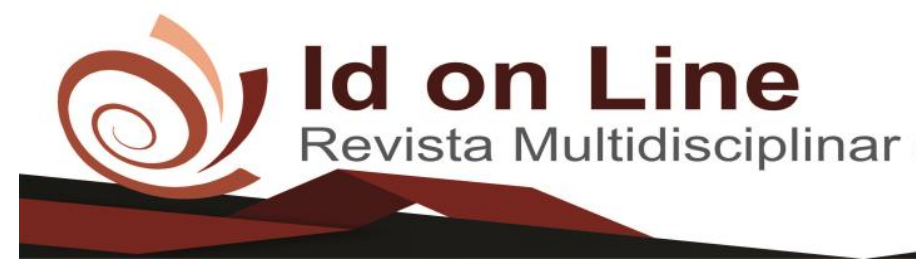

DOI: 10.14295/idonline.v13i47.2086

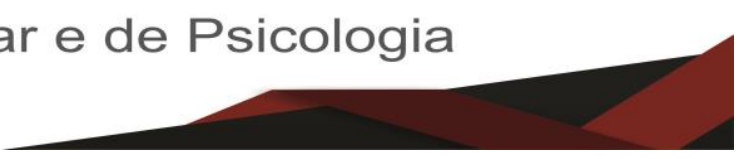

Artigo de Revisão

\title{
Clareamento Dental, Protocolo de aplicação em dentes vitais: Uma Revisão da Literatura
}

\author{
Felipe Rocha Carvalho ${ }^{1}$, Matheus Jacobina ${ }^{2}$, Bruno Bomfim Rocha ${ }^{3}$, Pablo Caique Leão ${ }^{4}$, \\ Fernando Clécio Santos do Carmo ${ }^{5}$, Erich Silva Almeida ${ }^{6}$
}

\begin{abstract}
Resumo: O objetivo do presente estudo foi analisar as evidências científicas sobre clareamento dental, os protocolos de aplicação em consultório e as melhores condutas clínicas. Foram realizada uma busca no banco de dados PubMed, Google Acadêmico e LiLacs para identificar as investigações disponíveis no período de 2009 a 2019. As seguintes palavras-chave foram utilizadas na busca: "CLAREAMENTO DENTAL" em português e também em inglês "TOOTH WHITENING". O clareamento dentário é um procedimento muito procurado na atualidade, com resultados estéticos satisfatórios quando feita a correta indicação e adequado uso. Entretanto, deve ser executado de forma cautelosa, tanto pelo profissional quanto pelo paciente, para proporcionar proteção pulpar e periodontal eficientes e não agredir tecidos importantes, evitando inflamações e sensibilidade pósoperatória, além de recidivas e manchamentos pós-operatórios.
\end{abstract}

Palavras-Chave: Odontologia. Clareamento Dental. Clareadores.

\section{Tooth Whitening, Vital Teeth Application Protocol: A Literature Review}

\begin{abstract}
The aim of the present study was to analyze the scientific evidence on teeth whitening, office application protocols and the best clinical management. We searched the PubMed, Google Scholar, and LiLacs database to identify the investigations available from 2009 to 2019. The following keywords were used in the search: "DENTAL CLEARING" in Portuguese as well as "TOOTH WHITENING". Tooth whitening is a much sought-after procedure today, with satisfactory aesthetic results when the correct indication and proper use are made. However, it should be performed cautiously by both the professional and the patient to provide efficient pulp and periodontal protection and not to damage important tissues, avoiding postoperative inflammation and sensitivity, as well as relapses and postoperative staining.
\end{abstract}

Keywords: Dentistry. Tooth whitening. Whitening

\footnotetext{
${ }^{1}$ Graduando em Odontologia, Faculdade Independente do Nordeste - FAINOR, Vitória da Conquista, BA, Brasil. Email: nxdfelipe@gmail.com

${ }^{2}$ Mestre em reabilitação oral pela faculdade USP/Bauru e professor de Odontologia, Faculdade Independente do Nordeste - FAINOR,

Vitória da Conquista, Ba, Brasil. Email: mjacobina@gmail.com

${ }^{3}$ Graduando em Odontologia, Faculdade Independente do Nordeste - FAINOR, Vitória da Conquista, BA, Brasil.

Email: rocha_bonfim@hotmail.com

${ }^{4}$ Graduando em Odontologia, Faculdade Independente do Nordeste - FAINOR, Vitória da Conquista, BA, Brasil.

Email: caiqueleao1@hotmail.com

${ }^{5}$ Graduando em Odontologia, Faculdade Independente do Nordeste - FAINOR, Vitória da Conquista, BA, Brasil.

Email: fernando18clecio@ hotmail.com

${ }^{6}$ Graduando em Odontologia, Faculdade Independente do Nordeste - FAINOR, Vitória da Conquista, BA, Brasil. Email: erich_almeida1@outlook.com
} 


\section{Introdução}

No final da década de 1980, várias empresas introduziram produtos para branqueamento dentário aplicados profissionalmente no mercado americano, devido ao aumento pela busca do sorriso bonito, com dentes mais brancos, seguindo os padrões estéticos da mídia (CAREY, 2014; MARSON; SENSI; REIS, 2008).

As colorações dentárias podem ser categorizadas em dois grupos principais: intrínseca e extrínseca. A coloração intrínseca, às vezes chamada de coloração interna, pode ser atribuída a fatores como genética, idade, uso de antibióticos, ingestão de altos níveis de flúor e distúrbios do desenvolvimento e podem ser iniciados antes da erupção do dente. A extrínseca, por sua vez, é em grande parte devido a fatores ambientais, incluindo o fumo, pigmentos em bebidas e alimentos, antibióticos e metais, como ferro ou cobre (CARDOSO et al., 2011; PERDIGÃO; LOGUÉRCIO; REIS, 2016).

O clareamento dentário é qualquer processo que deixe mais clara a cor de um dente. Pode ser realizado pela remoção física da mancha ou por uma reação química para clarear a cor do dente, retirando parcial ou totalmente a coloração extrínseca. O ingrediente ativo na maioria dos produtos de clareamento é o peróxido de hidrogênio (H2O2) (WIEGAND et al., 2008).

Atualmente, o tratamento clareador para dentes vitais é realizado com a aplicação, sobre os dentes, do peróxido de hidrogênio, em sua maioria, em concentrações que podem variar entre 30 a 38\% do ácido (BO et al., 2016; DEMARCO et al., 2016), com ou sem presença de fonte luminosa (ARDEKANI et al., 2018; CAREY, 2014). O clareamento em consultório com a supervisão de um profissional tem o resultado mais rápido comparado ao clareamento realizado em casa, visto que as concentrações do ácido é maior, fazendo com que o profissional fique atento a efeitos colaterais, como a sensibilidade dentária, ulceração nos tecidos moles bucais (MARSON; SENSI; REIS, 2008).

Com base no que foi descrito, a presente revisão teve como objetivo analisar as evidências científicas do clareamento dental, os protocolos de aplicação em consultório e as melhores condutas clínicas. 


\section{Métodos}

Foi realizado uma busca nos bancos de dados PubMed, Google Acadêmico e LiLacs para identificar as investigações disponíveis no período de 2009 a 2019. As seguintes palavras-chave foram utilizadas na busca: "Clareamento Dental” em português e em inglês “Tooth Whitening”. Foram excluídas das buscas publicações em revistas não científicas ou de avaliação Qualis Capes abaixo de B3, resumos e resumos expandidos, estudos experimentais não finalizados, teses, dissertações e monografias. Os artigos encontrados foram separados e adicionados no Mendeley para identificação de duplicatas, após a exclusão das duplicatas serão lidos os títulos para a primeira etapa da seleção dos artigos, em seguida a leitura dos resumos que tiveram caráter de exclusão ou permanência do manuscrito e por fim a leitura do artigo na íntegra para composição da revisão da literatura. Foi levado em consideração em cada artigo o tipo de clareamento e os tipos de produtos utilizados, respeitando as datas estipuladas para a pesquisa.

Fizeram parte deste estudo 81 artigos, que estão dispostos conforme a tabela 1 a seguir.

Tabela 1 - Artigos que fizeram parte do presente estudo.

\begin{tabular}{|c|c|c|c|}
\hline Autor (es) & Título & Periódico & Ano \\
\hline AKBARI, M. et al. & $\begin{array}{l}\text { Does at-home bleaching induce systemic oxidative stress } \\
\text { in healthy subjects? }\end{array}$ & $\begin{array}{l}\text { Australian Dental } \\
\text { Journal, v. } 62, \text { n. } 1, \mathrm{p} \text {. } \\
\text { 58-64. }\end{array}$ & 2017 \\
\hline ALVES, N. V. et al. & $\begin{array}{l}\text { Reabilitação Estética E Funcional Do Sorriso: Revisão } \\
\text { De Literatura. }\end{array}$ & $\begin{array}{l}\text { Revista Interfaces } \\
\text { saúde, humanas e } \\
\text { tecnologia., v. } 3 \text {, n. } 9 \\
\text { p. } 25-30 .\end{array}$ & 2016 \\
\hline ARDEKANI, Y. T. et al. & $\begin{array}{l}\text { Immediate clinical evaluation of a 940-nm diode laser- } \\
\text { assisted in-office bleaching technique. }\end{array}$ & $\begin{array}{l}\text { Lasers in Dental } \\
\text { Science, v. 2, n. 4, p. } \\
\text { 239-245. }\end{array}$ & 2018 \\
\hline BO, L. et al. & $\begin{array}{l}\text { Efeitos de clareamento de consultório no fluxo pulpar e } \\
\text { sensibilidade dentária: série de casos. }\end{array}$ & $\begin{array}{l}\text { Congresso } \\
\text { Odontológico do } \\
\text { Norte do Paraná, v. 5, } \\
\text { n. } 6, \text { p. } 21270, \text { Anais.. }\end{array}$ & 2016 \\
\hline $\begin{array}{l}\text { CANEPPELE, T. M. F. } \\
\text { et al. }\end{array}$ & $\begin{array}{l}\text { Influence of whitening gel application protocol on dental } \\
\text { color change. Scientific World Journal. }\end{array}$ & $\begin{array}{l}\text { Scientific World } \\
\text { Journal, v. } 2015\end{array}$ & 2015 \\
\hline CARDOSO, P. C. et al. & $\begin{array}{l}\text { Facetas Diretas de Resina Composta e Clareamento } \\
\text { Dental : Estratégias para Dentes Escurecidos Direct } \\
\text { Composite Resin Veneers and Dental Whitening: } \\
\text { Strategies for Discolored Teeth. }\end{array}$ & $\begin{array}{c}\text { Revista de } \\
\text { Odontologia } \\
\text { Brasileira Central, v. } \\
\text { 20, n. 55, p. 341-347. }\end{array}$ & 2011 \\
\hline CAREY, C. M. & Tooth whitening: What we now know. & $\begin{array}{l}\text { Journal of Evidence- } \\
\text { Based Dental } \\
\text { Practice, v. } 14, \text { n. } \\
\text { SUPPL., p. } 70-76 .\end{array}$ & 2014 \\
\hline CAREY, C. M. & Tooth whitening: What we now know. & $\begin{array}{l}\text { Journal of Evidence- } \\
\text { Based Dental } \\
\text { Practice, v. } 14, \mathrm{n} . \\
\end{array}$ & 2014 \\
\hline
\end{tabular}




\begin{tabular}{|c|c|c|c|}
\hline & & SUPPL., p. 70-76. & \\
\hline CASADO, B. G. S. et al. & $\begin{array}{l}\text { Efficacy of dental bleaching with whitening dentifrices: } \\
\text { A systematic review. }\end{array}$ & $\begin{array}{l}\text { International Journal } \\
\text { of Dentistry, v. } 2018 .\end{array}$ & 2018 \\
\hline $\begin{array}{l}\text { CHAN, W.; LYNCH, E.; } \\
\text { GROOTVELD, M. }\end{array}$ & $\begin{array}{l}\text { Summary of: Tooth-whitening activity of a novel home- } \\
\text { bleaching system utilising thermal diffusion: A } \\
\text { multifactorial simultaneous evaluation of efficacy at } \\
\text { cervical, body and incisal tooth sites. }\end{array}$ & $\begin{array}{l}\text { British Dental } \\
\text { Journal, v. } 212, \text { n. } 4 \\
\text { p. } 190-191 .\end{array}$ & 2012 \\
\hline CORCODEL, N. et al. & $\begin{array}{l}\text { Effect of enamel sealants on tooth bleaching and on the } \\
\text { color stability of the result. }\end{array}$ & $\begin{array}{l}\text { Odontology, v. } 105 \\
\text { n. } 2 \text {, p. } 155-161 .\end{array}$ & 2017 \\
\hline DA COSTA, J. B. et al. & $\begin{array}{l}\text { Comparison of at-home and in-office tooth whitening } \\
\text { using a novel shade guide. }\end{array}$ & $\begin{array}{l}\text { Operative Dentistry, } \\
\text { v. } 35, \text { n. } 4, \text { p. } 381- \\
388 .\end{array}$ & 2010 \\
\hline $\begin{array}{l}\text { DE VASCONCELOS, } \\
\text { A. A. M. et al. }\end{array}$ & $\begin{array}{l}\text { Tooth whitening with hydrogen/carbamide peroxides in } \\
\text { association with a CPP-ACP paste at different } \\
\text { proportions }\end{array}$ & $\begin{array}{l}\text {. Australian Dental } \\
\text { Journal, v. 57, n. 2, p. } \\
\text { 213-219. }\end{array}$ & 2012 \\
\hline DEMARCO, F. F. et al. & $\begin{array}{l}\text { Produtos de autocuidado para clareamento dental Self- } \\
\text { care products for tooth whitening. }\end{array}$ & $\begin{array}{l}\text { RFO, Passo Fundo, v. } \\
\text { 21, n. 1, p. } 143-149 .\end{array}$ & 2016 \\
\hline FEKRAZAD, R. et al. & $\begin{array}{l}\text { Comparison of laser and power bleaching techniques in } \\
\text { tooth color change }\end{array}$ & $\begin{array}{l}\text { Journal of Clinical } \\
\text { and Experimental } \\
\text { Dentistry, v. 9, n. 4, p. } \\
\text { e511-e515. }\end{array}$ & 2017 \\
\hline FIORILLO, L. et al. & $\begin{array}{l}\text { Dental Whitening Gels: Strengths and Weaknesses of an } \\
\text { Increasingly Used Method. Gels, v. 5, n. 3, p. 35, } 2019 .\end{array}$ & Gels, v. 5, n. 3, p. 35 . & 2019 \\
\hline $\begin{array}{ll}\text { GOLDBERG, } & \text { M.; } \\
\text { GROOTVELD, } & \text { M.; } \\
\text { LYNCH, E. } & \end{array}$ & $\begin{array}{l}\text { Undesirable and adverse effects of tooth-whitening } \\
\text { products: A review. }\end{array}$ & $\begin{array}{l}\text { Clinical Oral } \\
\text { Investigations, v. } 14 \\
\text { n. } 1, \text { p. } 1-10 .\end{array}$ & 2010 \\
\hline $\begin{array}{l}\text { GREENWALL-COHEN, } \\
\text { J. et al. }\end{array}$ & Tooth whitening for the under-18-year-old patient. & $\begin{array}{l}\text { British Dental } \\
\text { Journal, v. } 225, \text { n. } 1 \text {, } \\
\text { p. } 19-26 . \\
\end{array}$ & 2018 \\
\hline HENRY, R. K. et al. & $\begin{array}{l}\text { The effect of light on tooth whitening: A split-mouth } \\
\text { design. }\end{array}$ & $\begin{array}{l}\text { International } \\
\text { Journal of Dental } \\
\text { Hygiene, v. } 11, \mathrm{n} .2 \\
\text { p. } 151-154 .\end{array}$ & 2013 \\
\hline JIN, J. et al. & $\begin{array}{l}\text { Efficacy of tooth whitening with different calcium } \\
\text { phosphate-based formulations. }\end{array}$ & $\begin{array}{l}\text { European Journal of } \\
\text { Oral Sciences, v. } \\
121, \text { n. } 4 \text {, p. } 382-388 .\end{array}$ & 2013 \\
\hline JOINER, A. & Whitening toothpastes: A review of the literature. & $\begin{array}{l}\text { Journal of Dentistry, } \\
\text { v. } 38 \text {, n. SUPPL. 2, p. } \\
17-24 .\end{array}$ & 2010 \\
\hline JOINER, A.; LUO, W. & Tooth colour and whiteness: A review. [s.l.] & Elsevier Ltd, v. 67 & 2017 \\
\hline JPS, M.; ACT, J.; JA, R. & $\begin{array}{l}\text { Efeito Do Clareamento Dental Sobre Os Materiais } \\
\text { Restauradores Effect of Dental Bleaching on Restorative } \\
\text { Materials. v. 3, n. 2, p. 14-19, } 2009 .\end{array}$ & $\begin{array}{l}\text { Bleaching on } \\
\text { Restorative Materials. } \\
\text { v. 3, n. 2, p. 14-19. }\end{array}$ & 2009 \\
\hline KAEWPINTA, A. et al. & $\begin{array}{l}\text { Tooth whitening efficacy of pigmented rice gels } \\
\text { containing carbamide peroxide. }\end{array}$ & $\begin{array}{l}\text { Drug discoveries \& } \\
\text { therapeutics, v. } 12 \text {, } \\
\text { n. } 3, \text { p. } 126-132 . \\
\end{array}$ & 2018 \\
\hline KARADAS, M. & $\begin{array}{l}\text { Efficacy of whitening oral rinses and dentifrices on color } \\
\text { stability of bleached teeth. }\end{array}$ & $\begin{array}{l}\text { Acta Biomaterialia } \\
\text { Odontologica } \\
\text { Scandinavica, v. 1, n. } \\
\text { 1, p. 29-34. }\end{array}$ & 2015 \\
\hline $\begin{array}{l}\text { KARADAS, } \quad \text { M.; } \\
\text { HATIPOGLU, O. }\end{array}$ & $\begin{array}{l}\text { Efficacy of mouthwashes containing hydrogen peroxide } \\
\text { on tooth whitening. }\end{array}$ & $\begin{array}{l}\text { Scientific World } \\
\text { Journal, v. } 2015 .\end{array}$ & 2015 \\
\hline $\begin{array}{l}\text { KNEZOVIĆ } \\
\text { ZLATARIĆ, } \quad \text { D.; } \\
\text { ŽAGAR, M.; ILLEŠ, D. } \\
\text { A }\end{array}$ & $\begin{array}{l}\text { clinical study assessing the short-term efficacy of } \\
\text { combined in-office/at-home whitening treatment. }\end{array}$ & $\begin{array}{c}\text { Journal of Esthetic } \\
\text { and estorative } \\
\text { Dentistry, v. } 31 \text {, n. } 2 \text {, } \\
\text { p. } 140-146 .\end{array}$ & 2019 \\
\hline KOTHARI, S. et al. & $\begin{array}{l}\text { Vital bleaching and oral-health-related quality of life in } \\
\text { adults: A systematic review and meta-analysis. }\end{array}$ & $\begin{array}{c}\text { Journal of Dentistry, } \\
\text { v. } 84 \text {, n. December } \\
2018 \text {, p. } 22-29 .\end{array}$ & 2019 \\
\hline $\begin{array}{l}\text { KWON, S. R.; WERTZ, } \\
\text { P. W. }\end{array}$ & Review of the mechanism of tooth whitening. & $\begin{array}{c}\text { Journal of Esthetic } \\
\text { and Restorative } \\
\text { Dentistry, v. } 27 \text {, n. } 5 \text {, } \\
\text { p. } 240-257 .\end{array}$ & 2015 \\
\hline
\end{tabular}




\begin{tabular}{|c|c|c|c|}
\hline $\begin{array}{l}\text { LI, Y.; GREENWALL, } \\
\text { L. }\end{array}$ & $\begin{array}{l}\text { Safety issues of tooth whitening using peroxide-based } \\
\text { materials. }\end{array}$ & $\begin{array}{c}\text { British Dental } \\
\text { Journal, v. } 215, \text { n. } 1 \\
\text { p. } 29-34 . \\
\end{array}$ & 2013 \\
\hline MAIA, T. S. et al. & $\begin{array}{l}\text { Harmonização do sorriso com facetas diretas em resina } \\
\text { composta: relato de caso. }\end{array}$ & $\begin{array}{l}\text { Clín. int. j. braz. } \\
\text { dent, v. } 11, \text { n. } 4 \text {, p. } \\
392-401 .\end{array}$ & 2015 \\
\hline MATIS, B. A. et al. & White diet: Is it necessary during tooth whitening? & $\begin{array}{c}\text { Operative Dentistry, } \\
\text { v. } 40, \text { n. } 3, \text { p. } 235- \\
240 . \\
\end{array}$ & 2015 \\
\hline AKBARI, M. et al. & $\begin{array}{l}\text { Does at-home bleaching induce systemic oxidative stress } \\
\text { in healthy subjects? }\end{array}$ & $\begin{array}{l}\text { Australian Dental } \\
\text { Journal, v. } 62, \text { n. } 1 \\
\text { p. } 58-64 .\end{array}$ & 2017 \\
\hline ALVES, N. V. et al. & $\begin{array}{l}\text { Reabilitação Estética E Funcional Do Sorriso: Revisão } \\
\text { De Literatura. }\end{array}$ & $\begin{array}{l}\text { Revista Interfaces } \\
\text { saúde, humanas e } \\
\text { tecnologia., v. } 3 \text {, n. } 9, \\
\text { p. } 25-30 .\end{array}$ & 2016 \\
\hline ARDEKANI, Y. T. et al. & $\begin{array}{l}\text { Immediate clinical evaluation of a } 940-\mathrm{nm} \text { diode laser- } \\
\text { assisted in-office bleaching technique. }\end{array}$ & $\begin{array}{l}\text { Lasers in Dental } \\
\text { Science, v. 2, n. 4, p. } \\
\text { 239-245. }\end{array}$ & 2018 \\
\hline BO, L. et al. & $\begin{array}{l}\text { Efeitos de clareamento de consultório no fluxo pulpar e } \\
\text { sensibilidade dentária: série de casos. }\end{array}$ & $\begin{array}{c}\text { Congresso } \\
\text { Odontológico do } \\
\text { Norte do Paraná, } \\
\text { anais.. v. 5, n. 6, p. } \\
\text { 212-70. } \\
\end{array}$ & 2016 \\
\hline $\begin{array}{l}\text { CANEPPELE, T. M. F. } \\
\text { et al. }\end{array}$ & $\begin{array}{l}\text { Influence of whitening gel application protocol on dental } \\
\text { color change. }\end{array}$ & $\begin{array}{l}\text { Scientific World } \\
\text { Journal, v. } 2015 . \\
\end{array}$ & 2015 \\
\hline CARDOSO, P. C. et al. & $\begin{array}{l}\text { Facetas Diretas de Resina Composta e Clareamento } \\
\text { Dental: Estratégias para Dentes Escurecidos Direct } \\
\text { Composite Resin Veneers and Dental Whitening: } \\
\text { Strategies for Discolored Teeth. }\end{array}$ & $\begin{array}{c}\text { Revista de } \\
\text { Odontologia } \\
\text { Brasileira Central, v. } \\
\text { 20, n. 55, p. 341-347. }\end{array}$ & 2011 \\
\hline CAREY, C. M. & Tooth whitening: What we now know. & $\begin{array}{l}\text { Journal of Evidence- } \\
\text { Based Dental } \\
\text { Practice, v. 14, n. } \\
\text { SUPPL., p. 70-76. }\end{array}$ & 2014 \\
\hline CASADO, B. G. S. et al. & $\begin{array}{l}\text { Efficacy of dental bleaching with whitening dentifrices: } \\
\text { A systematic review. }\end{array}$ & $\begin{array}{c}\text { International } \\
\text { Journal of Dentistry, } \\
\text { v. } 2018 .\end{array}$ & 2018 \\
\hline $\begin{array}{l}\text { CHAN, W.; LYNCH, E.; } \\
\text { GROOTVELD, M. }\end{array}$ & $\begin{array}{l}\text { Summary of: Tooth-whitening activity of a novel home- } \\
\text { bleaching system utilising thermal diffusion: A } \\
\text { multifactorial simultaneous evaluation of efficacy at } \\
\text { cervical, body and incisal tooth sites. }\end{array}$ & $\begin{array}{l}\text { British Dental } \\
\text { Journal, v. } 212, \text { n. } 4 \\
\text { p. } 190-191 .\end{array}$ & 2012 \\
\hline CORCODEL, N. et al. & $\begin{array}{l}\text { Effect of enamel sealants on tooth bleaching and on the } \\
\text { color stability of the result. }\end{array}$ & $\begin{array}{l}\text { Odontology, v. } 105 \\
\text { n. 2, p. } 155-167 .\end{array}$ & 2017 \\
\hline DA COSTA, J. B. et al. & $\begin{array}{l}\text { Comparison of at-home and in-office tooth whitening } \\
\text { using a novel shade guide. }\end{array}$ & $\begin{array}{c}\text { Operative Dentistry, } \\
\text { v. } 35, \text { n. } 4 \text {, p. } 381- \\
388 .\end{array}$ & 2010 \\
\hline $\begin{array}{l}\text { DE VASCONCELOS, } \\
\text { A. A. M. et al. }\end{array}$ & $\begin{array}{l}\text { Tooth whitening with hydrogen/carbamide peroxides in } \\
\text { association with a CPP-ACP paste at different } \\
\text { proportions. }\end{array}$ & $\begin{array}{c}\text { Australian Dental } \\
\text { Journal, v. 57, n. 2, } \\
\text { p. 213-219. }\end{array}$ & 2012 \\
\hline DEMARCO, F. F. et al. & $\begin{array}{l}\text { Produtos de autocuidado para clareamento dental Self- } \\
\text { care products for tooth whitening. }\end{array}$ & $\begin{array}{l}\text { RFO, Passo Fundo, } \\
\text { v. } 21, \text { n. } 1, \text { p. } 143- \\
149 .\end{array}$ & 2016 \\
\hline FEKRAZAD, R. et al. & $\begin{array}{l}\text { Comparison of laser and power bleaching techniques in } \\
\text { tooth color change. }\end{array}$ & $\begin{array}{l}\text { Journal of Clinical } \\
\text { and Experimental } \\
\text { Dentistry, v. } 9, \text { n. } 4 \text {, } \\
\text { p. e } 511-\mathrm{e} 515 . \\
\end{array}$ & 2017 \\
\hline FIORILLO, L. et al. & $\begin{array}{l}\text { Dental Whitening Gels: Strengths and Weaknesses of an } \\
\text { Increasingly Used Method }\end{array}$ & $\begin{array}{l}\text { Gels, v. } 5, \text { n. } 3, \text { p. } \\
35 \text {. }\end{array}$ & 2019 \\
\hline $\begin{array}{ll}\text { GOLDBERG, } & \text { M.; } \\
\text { GROOTVELD, } & \text { M.; } \\
\text { LYNCH, E. } & \end{array}$ & $\begin{array}{l}\text { Undesirable and adverse effects of tooth-whitening } \\
\text { products: A review. }\end{array}$ & $\begin{array}{c}\text { Clinical Oral } \\
\text { Investigations, v. } 14, \\
\text { n. } 1, \text { p. } 1-10 .\end{array}$ & 2010 \\
\hline $\begin{array}{l}\text { GREENWALL-COHEN, } \\
\text { J. et al. }\end{array}$ & Tooth whitening for the under-18-year-old patient. & $\begin{array}{l}\text { British Dental } \\
\text { Journal, v. } 225, \text { n. } 1 \\
\text { p. } 19-26 .\end{array}$ & 2018 \\
\hline HENRY, R. K. et al. & The effect of light on tooth whitening: A split-mouth & International & 2013 \\
\hline
\end{tabular}




\begin{tabular}{|c|c|c|c|}
\hline & design. & $\begin{array}{c}\text { Journal of Dental } \\
\text { Hygiene, v. } 11, \text { n. } 2 \\
\text { p. } 151-154 . \\
\end{array}$ & \\
\hline JIN, J. et al. & $\begin{array}{l}\text { Efficacy of tooth whitening with different calcium } \\
\text { phosphate-based formulations. }\end{array}$ & $\begin{array}{l}\text { European Journal of } \\
\text { Oral Sciences, v. } \\
121, \text { n. } 4 \text {, p. 382-388. }\end{array}$ & 2013 \\
\hline JOINER, A. & Whitening toothpastes: A review of the literature. & $\begin{array}{l}\text { Journal of Dentistry, } \\
\text { v. } 38, \text { n. SUPPL. 2, p. } \\
\text { 17-24. }\end{array}$ & 2010 \\
\hline JOINER, A.; LUO, W. & Tooth colour and whiteness: A review. [s.l.] & Elsevier Ltd. v. 67 & 2017 \\
\hline JPS, M.; ACT, J.; JA, R. & $\begin{array}{l}\text { Efeito Do Clareamento Dental Sobre Os Materiais } \\
\text { Restauradores Effect of Dental }\end{array}$ & $\begin{array}{l}\text { Bleaching on } \\
\text { Restorative Materials. } \\
\text { v. 3, n. 2, p. 14-19. } \\
\end{array}$ & 2009 \\
\hline KAEWPINTA, A. et al. & $\begin{array}{l}\text { Tooth whitening efficacy of pigmented rice gels } \\
\text { containing carbamide peroxide. }\end{array}$ & $\begin{array}{c}\text { Drug discoveries \& } \\
\text { therapeutics, v. } 12 \\
\text { n. } 3 \text {, p. } 126-132 . \\
\end{array}$ & 2018 \\
\hline KARADAS, M. & $\begin{array}{l}\text { Efficacy of whitening oral rinses and dentifrices on color } \\
\text { stability of bleached teeth.. }\end{array}$ & $\begin{array}{l}\text { Acta Biomaterialia } \\
\text { Odontologica } \\
\text { Scandinavica, v. } 1, \mathrm{n} . \\
\text { 1, p. } 29-34 \\
\end{array}$ & 2015 \\
\hline $\begin{array}{ll}\text { KARADAS, } & \text { M.; } \\
\text { HATIPOGLU, O. } & \end{array}$ & $\begin{array}{l}\text { Efficacy of mouthwashes containing hydrogen peroxide } \\
\text { on tooth whitening }\end{array}$ & $\begin{array}{l}\text { Scientific World } \\
\text { Journal, v. } 2015 .\end{array}$ & 2015 \\
\hline $\begin{array}{l}\text { KNEZOVIĆ } \\
\text { ZLATARIĆ, } \quad \text { D.; } \\
\text { ŽAGAR, M.; ILLEŠ, D. }\end{array}$ & $\begin{array}{l}\text { A clinical study assessing the short-term efficacy of } \\
\text { combined in-office/at-home whitening treatment. }\end{array}$ & $\begin{array}{l}\text { Journal of Esthetic } \\
\text { and Restorative } \\
\text { Dentistry, v. } 31, \text { n. } 2 \\
\text { p. } 140-146 .\end{array}$ & 2019 \\
\hline KOTHARI, S. et al. & $\begin{array}{l}\text { Vital bleaching and oral-health-related quality of life in } \\
\text { adults: A systematic review and meta-analysis. }\end{array}$ & $\begin{array}{l}\text { Journal of Dentistry, } \\
\text { v. } 84 \text {, n. December } \\
\text { 2018, p. 22-29. } \\
\end{array}$ & 2019 \\
\hline $\begin{array}{l}\text { KWON, S. R.; WERTZ, } \\
\text { P. W. }\end{array}$ & Review of the mechanism of tooth whitening. & $\begin{array}{l}\text { Journal of Esthetic } \\
\text { and Restorative } \\
\text { Dentistry, v. } 27, \text { n. } 5 \text {, } \\
\text { p. } 240-257 .\end{array}$ & 2015 \\
\hline LI, Y.; GREENWALL, L & $\begin{array}{l}\text { Safety issues of tooth whitening using peroxide-based } \\
\text { materials. }\end{array}$ & $\begin{array}{c}\text { British Dental } \\
\text { Journal, v. } 215, \text { n. } 1 \\
\text { p. } 29-34 . \\
\end{array}$ & 2013 \\
\hline MAIA, T. S. et al. & $\begin{array}{l}\text { Harmonização do sorriso com facetas diretas em resina } \\
\text { composta: relato de caso. }\end{array}$ & $\begin{array}{l}\text { Clín. int. j. braz. } \\
\text { dent, v. } 11, \text { n. } 4 \text {, p. } \\
\quad 392-401 .\end{array}$ & 2015 \\
\hline $\begin{array}{l}\text { MARSON, } \quad \text { F. } \quad \text { C.; } \\
\text { SENSI, L. G.; REIS, R. }\end{array}$ & $\begin{array}{l}\text { Novo conceito na clareação dentária pela técnica no } \\
\text { consultório. }\end{array}$ & $\begin{array}{l}\text { Revista Dental press } \\
\text { Est, p. 55-66. } \\
\end{array}$ & 2008 \\
\hline MATIS, B. A. et al. & White diet: Is it necessary during tooth whitening? & $\begin{array}{l}\text { Operative Dentistry, } \\
\text { v. } 40, \text { n. } 3 \text {, p. } 235- \\
240 . \\
\end{array}$ & 2015 \\
\hline PARK, S. et al. & $\begin{array}{l}\text { The Effect of Delivery System and Light Activation on } \\
\text { Tooth Whitening Efficacy and Hydrogen Peroxide } \\
\text { Penetration. }\end{array}$ & $\begin{array}{l}\text { Journal of Esthetic } \\
\text { and Restorative } \\
\text { Dentistry, v. } 28, \text { n. } 5 \\
\text { p. } 313-320 .\end{array}$ & 2016 \\
\hline PAULA, A. B. et al. & $\begin{array}{l}\text { Effects on gastric mucosa induced by dental bleaching - } \\
\text { An experimental study with } 6 \% \text { hydrogen peroxide in } \\
\text { rats. }\end{array}$ & $\begin{array}{l}\text { Journal of Applied } \\
\text { Oral Science, v. } 23 \text {, } \\
\text { n. } 5, \text { p. } 497-507 . \\
\end{array}$ & 2015 \\
\hline PINTO, M. M. et al. & $\begin{array}{l}\text { Controlled clinical trial addressing teeth whitening with } \\
\text { hydrogen peroxide in adolescents: A 12-month follow- } \\
\text { up. }\end{array}$ & $\begin{array}{l}\text { Clinics, v. } 72, \mathrm{n} .3, \mathrm{p} . \\
\text { 161-170. }\end{array}$ & 2017 \\
\hline $\begin{array}{l}\text { REGINA SILVA, G. DA } \\
\text { et al. }\end{array}$ & $\begin{array}{l}\text { Tratamento Estético Com Diretas De Resina Composta- } \\
\text { Relato De Caso Early Treatment of Class Iii With Haas } \\
\text { Associated Expander To Face Mask of Petit-Relat of } \\
\text { Case Resumo. }\end{array}$ & $\begin{array}{l}\text { Revista UNINGÁ } \\
\text { Review, v. } 24 \text {, n. 3, p. } \\
\text { 27-31. }\end{array}$ & 2015 \\
\hline $\begin{array}{l}\text { REINHARDT, J. W. et } \\
\text { al. }\end{array}$ & $\begin{array}{l}\text { Effect of tooth-whitening procedures on stained } \\
\text { composite resins. }\end{array}$ & $\begin{array}{l}\text { Operative Dentistry, } \\
\text { v. } 44, \text { n. } 1, \text { p. } 65-75 .\end{array}$ & 2019 \\
\hline $\begin{array}{l}\text { RODRIGUES, R. B. et } \\
\text { al. }\end{array}$ & $\begin{array}{l}\text { Clareamento dentário associado à facetas Indiretas em } \\
\text { cerâmica: Abordagem minimamente invasiva . Tooth } \\
\text { bleaching associated with Ceramic Veneers: A } \\
\text { conservative approach.v. } 21, \text { n. } 59 \text {, p. } 520-525,2012 \text {. }\end{array}$ & $\begin{array}{l}\text { conservative approach } \\
\text {.v. } 21, \text { n. } 59, \text { p. } 520- \\
525 .\end{array}$ & 2012 \\
\hline
\end{tabular}




\begin{tabular}{|c|c|c|c|}
\hline $\begin{array}{l}\text { SERRAGLIO, C. R. et } \\
\text { al. }\end{array}$ & $\begin{array}{l}\text { Efficacy and safety of over-the-counter whitening strips } \\
\text { as compared to home-whitening with } 10 \% \text { carbamide } \\
\text { peroxide gel-systematic review of RCTs and } \\
\text { metanalysis. }\end{array}$ & $\begin{array}{l}\text { Clinical Oral } \\
\text { Investigations, v. } 20 \\
\text { n. } 1, \text { p. } 1-14 .\end{array}$ & 2016 \\
\hline SILVA, C. F. E et al. & $\begin{array}{l}\text { Restabelecimento da estética dentária por meio da } \\
\text { combinação de clareamento de consultório e caseiro. }\end{array}$ & $\begin{array}{l}\text { Revista da Associacao } \\
\text { Paulista de Cirurgioes } \\
\text { Dentistas, v. } 69, \text { n. } 4, \\
\text { p. } 364-368 .\end{array}$ & 2015 \\
\hline $\begin{array}{l}\text { SOETEMAN, G. D. et } \\
\text { al. }\end{array}$ & $\begin{array}{l}\text { Whitening dentifrice and tooth surface discoloration-a } \\
\text { systematic review and meta-analysis. }\end{array}$ & $\begin{array}{l}\text { International } \\
\text { Journal of Dental } \\
\text { Hygiene, v. } 16, \mathrm{n} .1 \\
\text { p. } 24-35 .\end{array}$ & 2018 \\
\hline SU, I. H. et al. & $\begin{array}{l}\text { Evaluating a Cobalt-Tetraphenylporphyrin Complex, } \\
\text { Functionalized with a Reduced Graphene Oxide } \\
\text { Nanocomposite, for Improved Tooth Whitening. }\end{array}$ & $\begin{array}{l}\text { Journal of Esthetic } \\
\text { and Restorative } \\
\text { Dentistry, v. } 28 \text {, n. } 5 \text {, } \\
\text { p. } 321-329 .\end{array}$ & 2016 \\
\hline $\begin{array}{l}\text { TAM, L. E.; KIM, N.; } \\
\text { DE SOUZA, G. M. }\end{array}$ & $\begin{array}{l}\text { Effect of tooth whitening strips on fatigue resistance and } \\
\text { flexural strength of bovine dentin in vitro. }\end{array}$ & $\begin{array}{l}\text { PLoS ONE, v. 12, n. } \\
\text { 3, p. } 1-11,2017 .\end{array}$ & 2017 \\
\hline TAO, D. et al. & $\begin{array}{l}\text { Tooth whitening evaluation of blue covarine containing } \\
\text { toothpastes. }\end{array}$ & $\begin{array}{l}\text { Journal of Dentistry, } \\
\text { v. } 67, \text { n. October, p. } \\
\text { S20-S24, } 2017 \text {. } \\
\end{array}$ & 2017 \\
\hline $\begin{array}{l}\text { THANATHORNWONG, } \\
\text { B.; SUEBNUKARN, S.; } \\
\text { OUIVIRACH, K. }\end{array}$ & $\begin{array}{l}\text { Decision support system for predicting color change after } \\
\text { tooth whitening. }\end{array}$ & $\begin{array}{l}\text { Computer Methods } \\
\text { and Programs in } \\
\text { Biomedicine, v. } 125, \\
\text { p. } 88-93,2016 . \\
\end{array}$ & 2016 \\
\hline $\begin{array}{ll}\text { THICKETT, } & \text { E.; } \\
\text { COBOURNE, M. T. } & \end{array}$ & $\begin{array}{l}\text { New developments in tooth whitening. The current status } \\
\text { of external bleaching in orthodontics. }\end{array}$ & $\begin{array}{l}\text { Journal of } \\
\text { Orthodontics, v. } 36 \\
\text { n. 3, p. } 194-201 \\
2009 .\end{array}$ & 2009 \\
\hline TORRES, C. R. G. et al. & $\begin{array}{l}\text { Efficacy of mouth rinses and toothpaste on tooth } \\
\text { whitening. }\end{array}$ & $\begin{array}{l}\text { Operative Dentistry, } \\
\text { v. } 38, \text { n. } 1, \text { p. } 57-62 \text {, } \\
2013 .\end{array}$ & 2013 \\
\hline WANG, C. et al. & An in vitro screening assay for dental stain cleaning. & $\begin{array}{l}\text { BMC Oral Health, v. } \\
17, \text { n. 1, p. } 1-10 \\
2017 .\end{array}$ & 2017 \\
\hline WESTLAND, S. et al. & $\begin{array}{l}\text { Investigation of the perceptual thresholds of tooth } \\
\text { whiteness. }\end{array}$ & $\begin{array}{l}\text { Journal of Dentistry, } \\
\text { v. } 67 \text {, n. July, p. S11- } \\
\text { S14, } 2017 \text {. }\end{array}$ & 2017 \\
\hline YOUNG, N. et al. & $\begin{array}{l}\text { A study of hydrogen peroxide chemistry and } \\
\text { photochemistry in tea stain solution with relevance to } \\
\text { clinical tooth whitening. }\end{array}$ & $\begin{array}{c}\text { Journal of Dentistry, } \\
\text { v. 40, n. SUPPL.2, p. } \\
\text { e11-e16, 2012 }\end{array}$ & 2012 \\
\hline
\end{tabular}

Fonte: Dados da pesquisa.

\section{Revisão da Literatura}

\section{Clareamento}

O clareamento dentário é a área da odontologia estética que sofreu melhorias drásticas nas últimas duas décadas. Atualmente, os pacientes preferem o clareamento dental em consultório, já que o efeito de clareamento pode ser alcançado principalmente após apenas uma visita. A técnica mais usada é o branqueamento em peróxido de hidrogênio a $35 \%$ em consultório (MAIA et al., 2015). O efeito clareador é resultado da degradação de moléculas 
orgânicas complexas responsáveis pela cor dos dentes em moléculas menos complexas, resultando em uma redução ou eliminação da descoloração (KWON; WERTZ, 2015; REGINA SILVA et al., 2015).

A resposta ao clareamento é afetada por vários fatores, incluindo aqueles relacionados ao paciente, idade do paciente e cor dos dentes da linha de base (RODRIGUES et al., 2012). Os resultados de pesquisas integradas mostram que, se a cor dos dentes é mantida constante, os pacientes mais jovens geralmente respondem melhor ao tratamento do que os pacientes mais velhos. Esse achado pode estar relacionado a diferenças na estrutura dentária com a idade do paciente (JPS; ACT; JA, 2009).

A eficácia do tratamento pode ser medida usando instrumentos e avaliação visual em relação a guias de cores comerciais, por exemplo guias de cores, dispositivos de captura de imagens digitais, colorímetros ou um espectrofotômetro. Esses guias de cores são o método mais comumente utilizado na prática clínica devido à sua baixo custo e facilidade de uso (SOARES et al., 2006).

O guia de cores clássicas da Vita foi um dos métodos usados anteriormente para identificar a cor dos dentes, na qual as cores dos dentes foram dispostas dependendo da luminosidade (B1, A1, B2, D2, A2, C1, C2, D4, A3, D3, B3, A3 .5, B4, C3, A4, C4) onde B1 é considerado o mais claro e C4 o mais escuro das guias de tonalidade (DEMARCO et al., 2016; SILVA et al., 2015). No entanto, a avaliação visual em relação a um guia de cores é altamente subjetiva, propensa ao viés do observador e muitas vezes difícil de combinar um dente natural com uma sombra (SILVA et al., 2015).

Um erro na determinação da tonalidade exata dos dentes pode ocorrer devido às limitações na seleção de cores com base no guia de tonalidades disponível, em comparação com a grande variedade possível de cores naturais dos dentes (REINHARDT et al., 2019). O olho humano, a luz ambiente, bem como as influências ambientais diretas e indiretas, são fatores que sempre influenciam subjetivamente a determinação da tonalidade dos dentes (PINTO et al., 2017). O spectroshade é uma nova tecnologia de medição baseada na de um espectrofotômetro, além da moderna funcionalidade de LED. Supera os desafios na determinação da tonalidade dos dentes, oferecendo resultados precisos e objetivos, sem quaisquer efeitos ambientais (FEKRAZAD et al., 2017).

Uma luz definida é passada para o dente e o espectro de remissão é medido. A cor foi determinada com os parâmetros CIELAB L* a * b*, em que as coordenadas L * (brilho) são medidas em uma escala de 0 (preto) a 100 (branco). O valor a * é uma medida de vermelhidão 
(+60) ou verde (-60). O valor b * é uma medida de amarelo (+60) ou azul (-60) (TAM; KIM; DE SOUZA, 2017).

\section{Agentes Clareadores}

A estrutura dental possui grande permeabilidade permitindo que os agentes clareadores/peróxido de hidrogênio que são veículos de radicais de oxigênio instáveis se difundam pelo esmalte e dentina, agindo sobre os pigmentos que causam a descoloração dental (GOLDBERG; GROOTVELD; LYNCH, 2010). Esses pigmentos são cadeias de alto peso molecular que vão sendo fragmentadas em cadeias menores até serem eliminadas total ou parcialmente da estrutura dental por um processo de difusão (JOINER; LUO, 2017).

Os agentes clareadores utilizados para clareamento de dentes vitais são o peróxido de hidrogênio e peróxido de carbamida, onde este último, quando em contato com a estrutura dental se dissocia em peróxido de hidrogênio (H2O2) e por sua vez em oxigênio $(\mathrm{O} 2)$, responsável pelo clareamento, e ureia que neutraliza o $\mathrm{Ph}$ do meio e amônia que aumenta a permeabilidade da estrutura dental (KWON; WERTZ, 2015; TAO et al., 2017).

O clareamento de dentes vitais pode ser dividido entre clareamento caseiro e clareamento realizado no consultório. O primeiro utiliza o peróxido de hidrogênio até 7,5\% e peróxido de carbamida de $10 \%$ a $22 \%$. 0 segundo utiliza peróxido de hidrogênio, em altas concentrações, de 35 é 38\% (JOINER; LUO, 2017).

Para o sucesso do tratamento clareador o importante é que se faça um diagnóstico preciso da etiologia da alteração da cor presente, executando uni adequado exame clínico e anamnese detalhada, por isso acontecer e saber identificar o manchamento dental é muito importante (SOETEMAN et al., 2018). Posteriormente ao diagnóstico segue o planejamento do caso que se torna um pouco limitado devido à dificuldade de prever os resultados imediatos e a longo prazo (MATIS et al., 2015).

Deve-se, também, esclarecer ao paciente a necessidade da troca de restaurações estéticas após o tratamento clareador, já que as restaurações não clareiam quando o gel clareador é utilizado como nos dentes. Para garantir um efetivo clareamento o paciente deve ser informado que o seu tratamento clareador caseiro pode durar de duas a seis semanas (JOINER; LUO, 2017). O tempo de tratamento sendo de 1 a 3 semanas com peróxido de carbamida à concentrações variadas, dependendo da etiologia do caso, o que pode desmotivar 
o paciente e esse preferir o clareamento no consultório que tem resultados iniciais mais expressivos (JOINER, 2010; JOINER; LUO, 2017).

\section{Clareamento Caseiro e de Consultório}

A procura pela estética dentária vem sendo verificada na odontologia há bastante tempo. Os pacientes estão cada vez mais exigentes com a aparência e com isso, cabe ao dentista atender a essas necessidades, proporcionando um sorriso mais harmônico, superando as expectativas desses pacientes que procuram dentes mais claros (ALVES et al., 2016).

Estudos revelam que em torno de $55 \%$ dos indivíduos de idades, gêneros e culturas diferentes têm avaliado a cor de seus dentes como insatisfatória, com ênfase às mulheres, que acreditam que dentes mais claros são fatores importantes para uma boa aparência (BO et al., 2016). Assim, a odontologia vem cumprindo um papel importante nesse processo desenvolvendo novas técnicas destinadas à promoção da autoestima das pessoas. Uma dessas técnicas é o clareamento dental, que consiste num procedimento menos invasivo, indicado na maioria dos casos (GREENWALL-COHEN et al., 2018).

$\mathrm{O}$ processo de clareamento consiste na aplicação de um gel clareador, à base de peróxido de carbamida ou peróxido de hidrogênio, sobre os dentes a serem branqueados (CANEPPELE et al., 2015; KARADAS; HATIPOGLU, 2015). Ambos os métodos devem ser indicados e supervisionados pelo cirurgião-dentista, sempre impondo limites ao paciente e esclarecendo-o sobre os efeitos colaterais, pois o tratamento é realizado de acordo com as características individuais de cada pessoa (THICKETT; COBOURNE, 2009).

Quanto à durabilidade de cor, o clareamento de consultório apresenta maior recidiva da cor anterior ao tratamento em comparação ao clareamento caseiro. Isso pode ser atribuído ao processo de desmineralização e remineralização constante durante o tratamento caseiro, que pode ser responsável pelo durabilidade e estabilidade maiores, ao contrário do de consultório onde a remineralização ocorre após o término completo do procedimento (FIORILLO et al., 2019; KNEZOVIĆ ZLATARIĆ; ŽAGAR; ILLEŠ, 2019). 


\section{Clareamento Caseiro}

Este é realizado pelo paciente por meio de moldeiras individualizadas, sendo mais empregadas pelos cirurgiões-dentistas as concentrações de peróxido de carbamida, que variam de $10 \%$ a $22 \%$, e as de peróxido de hidrogênio, que variam de $4 \%$ a $8 \%$ (AKBARI et al., 2017).

As moldeiras são confeccionadas à vácuo, sobre um modelo de gesso, com uma placa de acetato de aproximadamente $2 \mathrm{~mm}$ de espessura (DE VASCONCELOS et al., 2012). Esta por sua vez deve recobrir totalmente toda estrutura dental do arco, limitando apenas o contato com tecidos moles e palato, permitindo assim o máximo de conforto para o paciente e o mínimo de injúrias ao tecido gengival (WESTLAND et al., 2017).

O clareamento caseiro consiste em uma técnica de baixo custo e pouco tempo de consultório, sendo indicada para dentes com manchamentos médios. São realizadas uma consulta para confecção das moldeiras individuais, orientações sobre como manipular o gel clareador e informações sobre a utilização do produto clareador (MATIS et al., 2015).

Os agentes clareadores presentes no mercado são os peróxidos de carbamida $10 \%$ a $16 \%$ e peróxido de hidrogênio de $6 \%$ a $9,5 \%$, sendo sua posologia descrita em relação ao tempo de uso da moldeira, não apresentando diferença no resultado, quando comparados os produtos. Os mais utilizados são os peróxidos de carbamida a $10 \%$ e $16 \%$ (DE VASCONCELOS et al., 2012). O peróxido de carbamida é indicado para uso noturno pois libera ureia que melhora o $\mathrm{pH}$ e sua reação dura em média 8 horas. Já o peróxido de hidrogênio para o clareamento possui sua reação mais rápida e é recomendado em uso diurno e sua reação dura em média 4 horas.

Nos casos de pigmentação mais grave ou quando é necessário tratamento em curto prazo, recomenda-se gel a $16 \%$. Já o gel a $10 \%$ é indicado a dentes naturalmente amarelados, escurecidos pela idade, em casos de pigmentação normal ou quando a sensibilidade dental é mais intensa (KOTHARI et al., 2019).

O regime de tratamento pode variar de paciente para paciente. Pode-se optar pelo regime diurno ou noturno. Porém a decisão de clarear de dia ou de noite deve ser tomada mais pela disponibilidade paciente do que por evidência clínica. Em ambos os regimes é preferível que o paciente aplique o gel somente uma vez ao dia, pois a aplicação do produto mais que 
uma vez pode implicar em maior sensibilidade dental e irritação gengival (DA COSTA et al., 2010).

Também é mais aconselhado optar por um clareador menos concentrado e por um tempo maior de tratamento, uma a duas semanas extras, principalmente nos dentes inferiores, que são mais resistentes ao clareamento do que submeter o paciente a uma taxa de sensibilidade dental mais alta. Por isso a importância do profissional saber quando parar o tratamento clareador, já que a partir de um determinado momento ocorre o ponto de saturação, onde a perda da estrutura dental é maior que o ganho em termos de clareamento (KAEWPINTA et al., 2018).

É importante alertar o paciente da quantidade do material a ser aplicado na moldeira, restringindo-o a apenas uma gota sobre cada dente da moldeira. Durante o período em que estiver utilizando o gel, o paciente não deverá ingerir alimentos para que o agente clareador não sofra diluição nem contaminação (KARADAS, 2015). Quando alcançar a cor almejada, o paciente poderá interromper o tratamento ou o cirurgião-dentista poderá optar por continuar a aplicação do gel durante mais uma semana, visando estabilizar a cor obtida (CAREY, 2014).

As vantagens da técnica caseira incluem a utilização de agentes clareadores de baixa concentração, que podem ser aplicados, concomitantemente, em vários dentes e de substâncias fáceis de serem encontradas no mercado, além de não causar sequelas nos dentes nem em tecidos moles, quando utilizados adequadamente (DA COSTA et al., 2010).

\section{Clareamento no Consultório}

No tratamento realizado em consultório, é comum o uso de concentrações de peróxido de hidrogênio que variam de $35 \%$ a $40 \%$ e peróxido de carbamida a $37 \%$, controlado pelo dentista16. O produto mais utilizado nessa técnica é o peróxido de hidrogênio, sendo aplicado com o isolamento das margens gengivais para a proteção do paciente contra seus efeitos cáusticos (PARK et al., 2016). O clareamento no consultório tem com vantagem ser mais bem controlado pelo profissional e rapidez de resultados, mas suas principais desvantagens são a maior sensibilidade dental e maior desgaste da superfície dental, já que a concentração do agente clareador é maior (HENRY et al., 2013).

A técnica operatória deve ser seguida corretamente. Diagnóstico do grau de escurecimento dental; registro da cor; planejamento do caso com o paciente; óculos de 
proteção no paciente, profissional e auxiliar; proteção da gengiva do paciente com produto insolúvel em água, como Omncilon-A em orabase (Bristil-Myers Squibb Brasil) (CHAN; LYNCH; GROOTVELD, 2012). Não deve anestesiar o paciente já que ele pode controlar e regular a sensibilidade dental, avaliando possíveis problemas no isolamento e a quantidade de aplicações do produto (KWON; WERTZ, 2015). Isolamento absoluto adequado; profilaxia com pedra-pomes e água ou pasta profilática sem óleo; manipulação do agente clareador e aplicação nas faces vestibular e proximais dos dentes (LI; GREENWALL, 2013).

A técnica de clareamento em consultório, tem como principal vantagem a possibilidade de dispensar o uso da moldeira causando menor desconforto ao paciente, para atingir o grau de satisfação de clareamento do paciente é necessário o número de 4 a 6 sessões em dentes que possuem tons mais escuros, com intervalos semanais entre cada sessão, ou seja, é necessário 6 semanas com aplicações de até 45 minutos, dependendo do agente clareador utilizado e da resposta do paciente quanto a sensibilidade provocada por esses géis e pela alteração de coloração dos elementos dentais (CASADO et al., 2018; KARADAS, 2015).

Essa técnica não depende da colaboração do paciente em casa, conferindo ao cirurgião-dentista mais controle sobre os locais de aplicação do gel clareador. Porém, o paciente pode relatar mais sensibilidade comparada à técnica de clareamento caseira, uma vez que a concentração de hidrogênio puro chega à polpa com mais disponibilidade que o peróxido de carbamida e o uso da luz aumenta a temperatura da polpa, elevando o nível de sensibilidade do paciente, além de ser necessário mais tempo de atendimento clínico (PAULA et al., 2015; REINHARDT et al., 2019).

Esse tipo de clareamento não é recomendado a pacientes com condições précancerígenas, fumantes, gestantes, lactantes e àqueles que apresentam irritações gengivais severas. O clareamento de consultório pode apresentar resultados possíveis de serem observados em uma a duas sessões, mas é possível que haja incidência de sensibilidade dental (SERRAGLIO et al., 2016).

Quanto à utilização da fonte de luz no clareamento de consultório, é utilizado para aumentar a temperatura do gel, acelerando a reação, mas pode ocasionar desconforto aos tecidos moles e agravos à polpa. A relação do cirurgião-dentista com o paciente deve ser clara no sentido de deixá-lo ciente de que poderá haver sensibilidade dental e gengival (JIN et al., 2013). Nos casos em que houver restaurações, ele deverá ser alertado sobre possíveis alterações. Durante o tratamento, o paciente deverá evitar o consumo de corantes, além de não 
poder fumar no período de tratamento, para, assim, obter resultados satisfatórios (CORCODEL et al., 2017; TORRES et al., 2013).

Para chegar a resultados satisfatórios, em algumas situações, poderão ser empregadas até seis sessões, respeitando um intervalo de sete dias. Há estudos nos quais a utilização de peróxido de hidrogênio a 35\%, durante 45 minutos, reduziu sua eficácia e causou hipersensibilidade, no entanto, quando o gel ficou em contato com o dente durante 15 minutos, em três aplicações, a sensibilidade causou menos incômodo (THANATHORNWONG; SUEBNUKARN; OUIVIRACH, 2016; YOUNG et al., 2012).

Porém, hoje, no mercado, há produtos mais novos, também compostos de peróxido de hidrogênio a 35\%, que não necessitam da troca do gel clareador da superfície do dente, podendo agir durante 50 minutos (SU et al., 2016; WANG et al., 2017).

\section{Considerações Finais}

O clareamento dentário é um procedimento muito procurado na atualidade, com resultados estéticos satisfatórios quando feita a correta indicação e adequado uso. Entretanto, deve ser executado de forma cautelosa, tanto pelo profissional quanto pelo paciente, para proporcionar proteção pulpar e periodontal eficientes e não agredir tecidos importantes, evitando inflamações e sensibilidade pós-operatória, além de recidivas e manchamentos pósoperatórios.

\section{Referências}

AKBARI, M. et al. Does at-home bleaching induce systemic oxidative stress in healthy subjects? Australian Dental Journal, v. 62, n. 1, p. 58-64, 2017.

ALVES, N. V. et al. Reabilitação Estética E Funcional Do Sorriso: Revisão De Literatura. Revista Interfaces saúde, humanas e tecnologia., v. 3, n. 9, p. 25-30, 2016.

ARDEKANI, Y. T. et al. Immediate clinical evaluation of a 940-nm diode laser-assisted inoffice bleaching technique. Lasers in Dental Science, v. 2, n. 4, p. 239-245, 2018.

BO, L. et al. Efeitos de clareamento de consultório no fluxo pulpar e sensibilidade dentária: série de casos. Congresso Odontológico do Norte do Paraná, anais.. v. 5, n. 6, p. 21270, 2016. 
CANEPPELE, T. M. F. et al. Influence of whitening gel application protocol on dental color change. Scientific World Journal, v. 2015, 2015.

CARDOSO, P. C. et al. Facetas Diretas de Resina Composta e Clareamento Dental: Estratégias para Dentes Escurecidos Direct Composite Resin Veneers and Dental Whitening : Strategies for Discolored Teeth. Revista de Odontologia Brasileira Central, v. 20, n. 55, p. 341-347, 2011.

CAREY, C. M. Tooth whitening: What we now know. Journal of Evidence-Based Dental Practice, v. 14, n. SUPPL., p. 70-76, 2014.

CASADO, B. G. S. et al. Efficacy of dental bleaching with whitening dentifrices: A systematic review. International Journal of Dentistry, v. 2018, 2018.

CHAN, W.; LYNCH, E.; GROOTVELD, M. Summary of: Tooth-whitening activity of a novel home-bleaching system utilising thermal diffusion: A multifactorial simultaneous evaluation of efficacy at cervical, body and incisal tooth sites. British Dental Journal, v. 212, n. 4, p. 190-191, 2012.

CORCODEL, N. et al. Effect of enamel sealants on tooth bleaching and on the color stability of the result. Odontology, v. 105, n. 2, p. 155-161, 2017.

DA COSTA, J. B. et al. Comparison of at-home and in-office tooth whitening using a novel shade guide. Operative Dentistry, v. 35, n. 4, p. 381-388, 2010.

DE VASCONCELOS, A. A. M. et al. Tooth whitening with hydrogen/carbamide peroxides in association with a CPP-ACP paste at different proportions. Australian Dental Journal, v. 57, n. 2, p. 213-219, 2012.

DEMARCO, F. F. et al. Produtos de autocuidado para clareamento dental Self-care products for tooth whitening. RFO, Passo Fundo, v. 21, n. 1, p. 143-149, 2016.

FEKRAZAD, R. et al. Comparison of laser and power bleaching techniques in tooth color change. Journal of Clinical and Experimental Dentistry, v. 9, n. 4, p. e511-e515, 2017.

FIORILLO, L. et al. Dental Whitening Gels: Strengths and Weaknesses of an Increasingly Used Method. Gels, v. 5, n. 3, p. 35, 2019.

GOLDBERG, M.; GROOTVELD, M.; LYNCH, E. Undesirable and adverse effects of toothwhitening products: A review. Clinical Oral Investigations, v. 14, n. 1, p. 1-10, 2010.

GREENWALL-COHEN, J. et al. Tooth whitening for the under-18-year-old patient. British Dental Journal, v. 225, n. 1, p. 19-26, 2018.

HENRY, R. K. et al. The effect of light on tooth whitening: A split-mouth design. International Journal of Dental Hygiene, v. 11, n. 2, p. 151-154, 2013.

JIN, J. et al. Efficacy of tooth whitening with different calcium phosphate-based formulations. European Journal of Oral Sciences, v. 121, n. 4, p. 382-388, 2013. 
JOINER, A. Whitening toothpastes: A review of the literature. Journal of Dentistry, v. 38, n. SUPPL. 2, p. 17-24, 2010.

JOINER, A.; LUO, W. Tooth colour and whiteness: A review. [s.1.] Elsevier Ltd, 2017. v. 67

JPS, M.; ACT, J.; JA, R. Efeito Do Clareamento Dental Sobre Os Materiais Restauradores Effect of Dental Bleaching on Restorative Materials. v. 3, n. 2, p. 14-19, 2009.

KAEWPINTA, A. et al. Tooth whitening efficacy of pigmented rice gels containing carbamide peroxide. Drug discoveries \& therapeutics, v. 12, n. 3, p. 126-132, 2018.

KARADAS, M. Efficacy of whitening oral rinses and dentifrices on color stability of bleached teeth. Acta Biomaterialia Odontologica Scandinavica, v. 1, n. 1, p. 29-34, 2015.

KARADAS, M.; HATIPOGLU, O. Efficacy of mouthwashes containing hydrogen peroxide on tooth whitening. Scientific World Journal, v. 2015, 2015.

KNEZOVIĆ ZLATARIĆ, D.; ŽAGAR, M.; ILLEŠ, D. A clinical study assessing the shortterm efficacy of combined in-office/at-home whitening treatment. Journal of Esthetic and Restorative Dentistry, v. 31, n. 2, p. 140-146, 2019.

KOTHARI, S. et al. Vital bleaching and oral-health-related quality of life in adults: A systematic review and meta-analysis. Journal of Dentistry, v. 84, n. December 2018, p. 22 29, 2019.

KWON, S. R.; WERTZ, P. W. Review of the mechanism of tooth whitening. Journal of Esthetic and Restorative Dentistry, v. 27, n. 5, p. 240-257, 2015.

LI, Y.; GREENWALL, L. Safety issues of tooth whitening using peroxide-based materials. British Dental Journal, v. 215, n. 1, p. 29-34, 2013.

MAIA, T. S. et al. Harmonização do sorriso com facetas diretas em resina composta: relato de caso. Clín. int. j. braz. dent, v. 11, n. 4, p. 392-401, 2015.

MARSON, F. C.; SENSI, L. G.; REIS, R. Novo conceito na clareação dentária pela técnica no consultório. Revista Dental press Est, p. 55-66, 2008.

MATIS, B. A. et al. White diet: Is it necessary during tooth whitening? Operative Dentistry, v. 40, n. 3, p. 235-240, 2015.

PARK, S. et al. The Effect of Delivery System and Light Activation on Tooth Whitening Efficacy and Hydrogen Peroxide Penetration. Journal of Esthetic and Restorative Dentistry, v. 28, n. 5, p. 313-320, 2016.

PAULA, A. B. et al. Effects on gastric mucosa induced by dental bleaching - An experimental study with 6\% hydrogen peroxide in rats. Journal of Applied Oral Science, v. 23, n. 5, p. 497-507, 2015.

PERDiGÃO, J.; LOGUÉRCIO, A. D.; REIS, A. At-Home Tooth Whitening. [s.l: s.n.]. 
PINTO, M. M. et al. Controlled clinical trial addressing teeth whitening with hydrogen peroxide in adolescents: A 12-month follow-up. Clinics, v. 72, n. 3, p. 161-170, 2017.

REGINA SILVA, G. DA et al. Tratamento Estético Com Diretas De Resina Composta-Relato De Caso Early Treatment of Class Iii With Haas Associated Expander To Face Mask of PetitRelat of Case Resumo. Revista UNINGÁ Review, v. 24, n. 3, p. 27-31, 2015.

REINHARDT, J. W. et al. Effect of tooth-whitening procedures on stained composite resins. Operative Dentistry, v. 44, n. 1, p. 65-75, 2019.

RODRIGUES, R. B. et al. Clareamento dentário associado à facetas Indiretas em cerâmica : Abordagem minimamente invasiva . Tooth bleaching associated with Ceramic Veneers: A conservative approach . v. 21, n. 59, p. 520-525, 2012.

SERRAGLIO, C. R. et al. Efficacy and safety of over-the-counter whitening strips as compared to home-whitening with $10 \%$ carbamide peroxide gel—systematic review of RCTs and metanalysis. Clinical Oral Investigations, v. 20, n. 1, p. 1-14, 2016.

SILVA, C. F. E et al. Restabelecimento da estética dentária por meio da combinação de clareamento de consultório e caseiro. Revista da Associacao Paulista de Cirurgioes Dentistas, v. 69, n. 4 , p. $364-368,2015$.

SOARES, C. J. et al. Avaliação clínica de clareamento caseiro com gel de peróxido de carbamida industrializado e manipulado em farmácia. Rev Odontol UNESP, v. 35, n. 1, p. 60-74, 2006.

SOETEMAN, G. D. et al. Whitening dentifrice and tooth surface discoloration-a systematic review and meta-analysis. International Journal of Dental Hygiene, v. 16, n. 1, p. 24-35, 2018.

SU, I. H. et al. Evaluating a Cobalt-Tetraphenylporphyrin Complex, Functionalized with a Reduced Graphene Oxide Nanocomposite, for Improved Tooth Whitening. Journal of Esthetic and Restorative Dentistry, v. 28, n. 5, p. 321-329, 2016.

TAM, L. E.; KIM, N.; DE SOUZA, G. M. Effect of tooth whitening strips on fatigue resistance and flexural strength of bovine dentin in vitro. PLoS ONE, v. 12, n. 3, p. 1-11, 2017.

TAO, D. et al. Tooth whitening evaluation of blue covarine containing toothpastes. Journal of Dentistry, v. 67, n. October, p. S20-S24, 2017.

THANATHORNWONG, B.; SUEBNUKARN, S.; OUIVIRACH, K. Decision support system for predicting color change after tooth whitening. Computer Methods and Programs in Biomedicine, v. 125, p. 88-93, 2016.

THICKETT, E.; COBOURNE, M. T. New developments in tooth whitening. The current status of external bleaching in orthodontics. Journal of Orthodontics, v. 36, n. 3, p. 194201, 2009.

TORRES, C. R. G. et al. Efficacy of mouth rinses and toothpaste on tooth whitening. Operative Dentistry, v. 38, n. 1, p. 57-62, 2013. 
WANG, C. et al. An in vitro screening assay for dental stain cleaning. BMC Oral Health, v. 17, n. 1, p. 1-10, 2017.

WESTLAND, S. et al. Investigation of the perceptual thresholds of tooth whiteness. Journal of Dentistry, v. 67, n. July, p. S11-S14, 2017.

WIEGAND, A. et al. 12-Month color stability of enamel, dentine, and enamel - Dentine samples after bleaching. Clinical Oral Investigations, v. 12, n. 4, p. 303-310, 2008.

YOUNG, N. et al. A study of hydrogen peroxide chemistry and photochemistry in tea stain solution with relevance to clinical tooth whitening. Journal of Dentistry, v. 40, n. SUPPL.2, p. e11-e16, 2012.

\section{Como citar este artigo (Formato ABNT):}

CARVALHO, Felipe Rocha; JACOBINA, Matheus; ROCHA, Bruno Bomfim; LEÃO, Pablo Caique; CARMO, Fernando Clécio Santos do; ALMEIDA, Erich Silva. Clareamento Dental, Protocolo de aplicação em dentes vitais: Uma Revisão da Literatura. Id on Line Rev.Mult. Psic., Outubro/2019, vol.13, n.47, p. 857-874. ISSN: 1981-1179.

Recebido: 15/10/2019;

Aceito: $21 / 10 / 2019$. 